\title{
Entrelacs
}

Cinéma et audiovisuel

$10 \mid 2013$

Le Toucher

\section{La place du toucher dans une politique du regard: Touch, Rise and Fall d'Aernout Mik}

\section{Mathilde Roman}

\section{(2) OpenEdition}

\section{Journals}

Édition électronique

URL : http://journals.openedition.org/entrelacs/536

DOI : 10.4000/entrelacs.536

ISSN : 2261-5482

Éditeur

Éditions Téraèdre

Référence électronique

Mathilde Roman, "La place du toucher dans une politique du regard: Touch, Rise and Fall d'Aernout Mik», Entrelacs [En ligne], 10 | 2013, mis en ligne le 12 septembre 2013, consulté le 01 mai 2019. URL : http://journals.openedition.org/entrelacs/536 ; DOI : 10.4000/entrelacs.536

Ce document a été généré automatiquement le 1 mai 2019.

Tous droits réservés 


\title{
La place du toucher dans une politique du regard: Touch, Rise and Fall d'Aernout Mik
}

\author{
Mathilde Roman
}

1 Dans les sociétés occidentales et plus particulièrement européennes, le toucher est globalement refoulé, géré par des normes de bienséance et par des répartitions architecturales et urbanistiques qui maintiennent une distance entre les intimités. Tout est fait pour éviter que les corps ne se touchent. Certaines situations contraignent néanmoins l'individu à un contact avec l'autre. Aernout Mik, artiste néerlandais né en 1962, s'intéresse à ces moments où les frontières de l'espace intime sont franchies, souvent en réponse à l'adversité, au danger, au sentiment de la catastrophe. Dans plusieurs de ses œuvres il filme des corps qui abandonnent leurs tenues, explorant des états limites où les individus ne tiennent plus le face-à-face avec le monde et autrui, où les corps s'affaissent et font masse, s'adressant au spectateur dans sa physicalité.

\section{Le corps à corps : une étape du vivre-ensemble?}

2 Pendant ses études dans les années 1990, Aernout Mik est très lié à son professeur de sculpture, l'artiste britannique Thom Puckey qui réalise dans les années 1970 au sein du duo « Reindeer Werk» (avec Dirk Larsen) des performances violentes, autoréférentielles et autoagressives, souhaitant amener les spectateurs dans un processus de conscientisation de leurs comportements conditionnés. Cette dimension performative où un corps renvoie avant tout à lui-même, où un individu s'affirme dans son isolement psychique, traverse l'œuvre d'Aernout Mik, et se retrouve radicalisée par l'utilisation du médium de la vidéo, que l'artiste utilise exclusivement. Ses vidéos mettent en scène des acteurs dans des postures physiques particulières. Elles explorent des normes du corps social par des déplacements de gestes, par des étirements temporels, par des processus de répétition et de décontextualisation. Son œuvre est traversée par la question du vivre- 
ensemble, interrogeant la manière dont les corps individuels s'articulent pour former une société. Le titre de l'exposition au Jeu de Paume, "Communitas "1, se référait à un concept important développé par Victor Turner, anthropologue britannique (1920-1983) qui désignait ainsi un moment particulier et transitoire où les individus se retrouvent dans une égalité sociale provisoire libérant une grande spontanéité. C'est une étape non structurelle dans la construction d'une société où la sensation du vivre-ensemble est très forte, et qui va ensuite être perdue avec la mise en place d'une structure sociale. Le communitas est un moment utopique et temporaire qui viendrait donc en amont des sociétés. Sans être nostalgique d'un paradis perdu, l'œuvre d'Aernout Mik questionne cette étape liminale en mettant en scène des individus physiquement regroupés, en contact tactilement les uns avec les autres, mais qui sont toujours isolés. Ils partagent un espace, un cadre, mais ce partage révèle d'autant mieux leur solitude existentielle : ils ne disposent même pas du langage pour installer un dialogue.

Aernout Mik écrit des scénarios joués de manière très performative par des acteurs souvent non professionnels. Chaque fois, les situations sont vaguement reconnaissables sans qu'il ne soit pourtant possible de les nommer précisément. Le spectateur découvre des scènes intrigantes dont il identifie certains signes, comme des corps cherchant la fuite ou installés pour un sitting, sans pouvoir les relier de manière certaine à des événements réels. De manière récurrente, les individus filmés par Aernout Mik sont entraînés dans des expériences de groupe où les corps se touchent et s'exposent au regard, dessinent une foule aux mouvements chorégraphiques sans jamais se constituer comme groupe. Les consciences s'engluent et les repères s'effacent tandis que les corps s'approchent. L'exploration de cet état liminaire à la société est associée à un rabaissement de l'individu dont la personnalité est neutralisée avant d'être revalorisée lors de la construction d'un ordre collectif ${ }^{2}$. Ce sont ainsi des identités tenues à distance qu'Aernout Mik filme, dépossédées de leur humanité et de la parole.

Fig. 1 : Aernout Mik, Organic Escalator, 2000. Single channel video installation, video still courtesy carlier | gebauer, Berlin

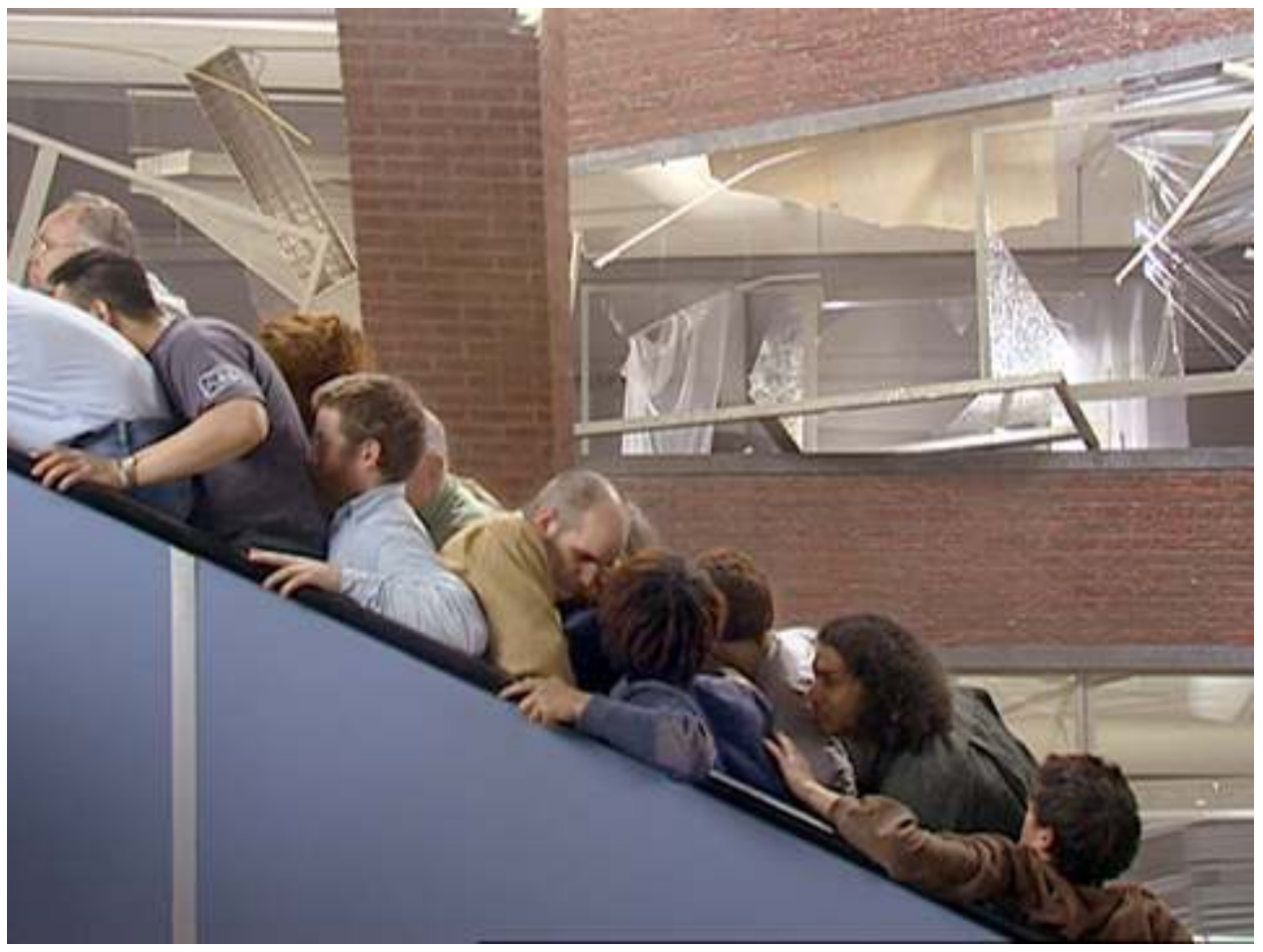


Dans Organic escalator (2000) par exemple, le visiteur pénètre un espace de projection étroit où une image de plain-pied montre des hommes et des femmes cherchant à gravir un escalator. Le climat de catastrophe se lit dans les postures et se devine surtout grâce au décor projeté derrière eux qui donne à voir l'écroulement d'une façade : bois, tissu, plaques de plâtres s'effondrent. La structure de projection en placoplâtre tremble elleaussi, infiltrant la fiction dans l'espace réel par un dispositif immersif. L'escalier est pris d'assaut par des corps nombreux ne parvenant pas à avancer, cherchant à s'extirper de là sans cris ni violence. Ils se touchent, sans agressivité mais sans non plus communiquer. Leur proximité physique contraste avec un isolement psychique ; chacun est seul dans sa tentative de fuite, bien que leurs mouvements ne puissent s'autonomiser de ceux qui les précèdent. Une impression de lenteur et de calme apparent domine la scène, comme en témoignent souvent les rescapés de tremblements de terre ou d'attentats ${ }^{3}$. L'un d'entre eux est étrangement tourné vers la descente, mais son corps est pris dans un flux global qui l'empêche de s'autonomiser et donne à l'ensemble une dimension chorégraphique. Le spectateur est facilement happé par cette scène décontextualisée et étirée temporellement par la mise en boucle.

Fig 2 : Aernout Mik, Glutinosity, 2001, single screen video installation, video still, courtesy carlier I gebauer

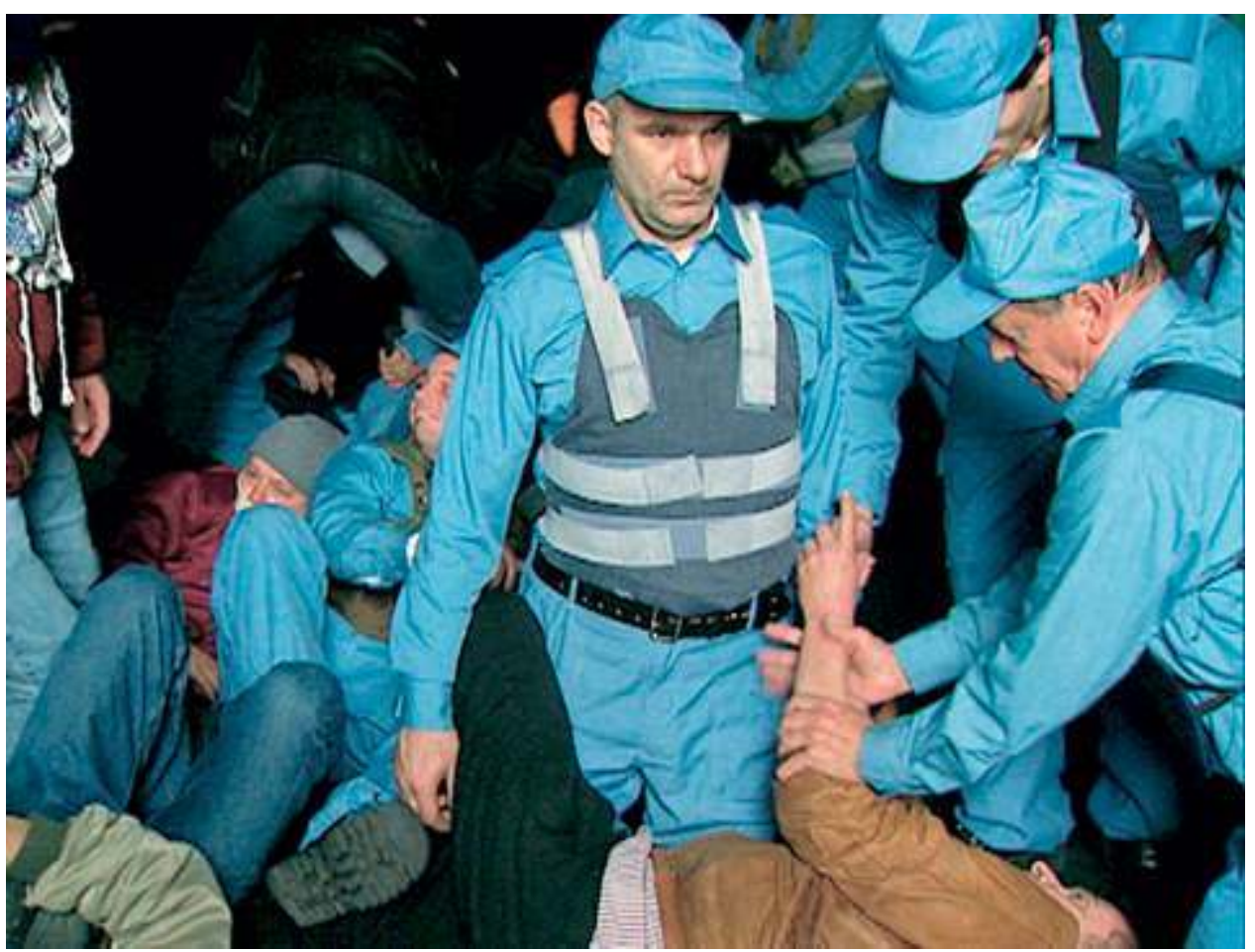

Dans Glutinosity (2001), Aernout Mik filme en gros plan une foule de corps entassés divisés en deux groupes : ceux qui affichent une résistance en s'enracinant au sol, et ceux qui cherchent à les faire sortir du cadre, à les déplacer. Une situation qui fait écho aux luttes entre manifestants et forces de l'ordre, où, comme le souligne cette œuvre, des corps passifs exprimant une volonté de résistance individuelle contre l'ordre collectif s'opposent à des corps s'agitant pour protéger bien souvent un immobilisme politique. Mais bien vite les rôles s'inversent, empêchant une lecture politique trop littérale. Aernout Mik s'intéresse aux images médiatiques et à la réduction signifiante qu'elles opèrent. A contrario, ses œuvres complexifient notre lecture en perturbant les repères. 
Ainsi dans Glutinosity, la relation dominants/ dominés s'inverse à un moment, et la présentation en boucle permet qu'il n'y ait ni vainqueurs ni vaincus.

Dans Training Ground (2006) encore, Aernout Mik filme une séance d'entraînement où des gardes en uniforme doivent interpeller des immigrants irréguliers. Les gestes bien connus se succèdent - mise à terre, fouille au corps - puis de manière inattendue les gardes s'assoient eux aussi, désemparés, voire même angoissés, tandis qu'un immigrant s'éloigne tranquillement. Les vidéos d'Aernout Mik n'offrent pas le refuge de lectures univoques teintées d'idéologie. Le spectateur est confronté à une vision du réel revendiquant l'enchevêtrement.

7 Dans ces trois œuvres, les corps se touchent et pourtant ne se parlent pas. Loin de l'horizon communautaire décrit par Victor Turner, l'œuvre d'Aernout Mik engage une réflexion sur la place réservée au toucher dans les sociétés contemporaines. Marginalisé dans les relations humaines, cantonné dans la sphère de l'intimité, le toucher est déconnecté de l'expérience de la rencontre entre deux êtres. De manière très révélatrice, c'est dans un contexte de suspicion que le sens du toucher est utilisé, lors des contrôles dans les aéroports. Aernout Mik a ainsi consacré une de ses pièces à ces pratiques tactiles de la surveillance.

\section{Le toucher et la vision dans la gestion de la surveillance}

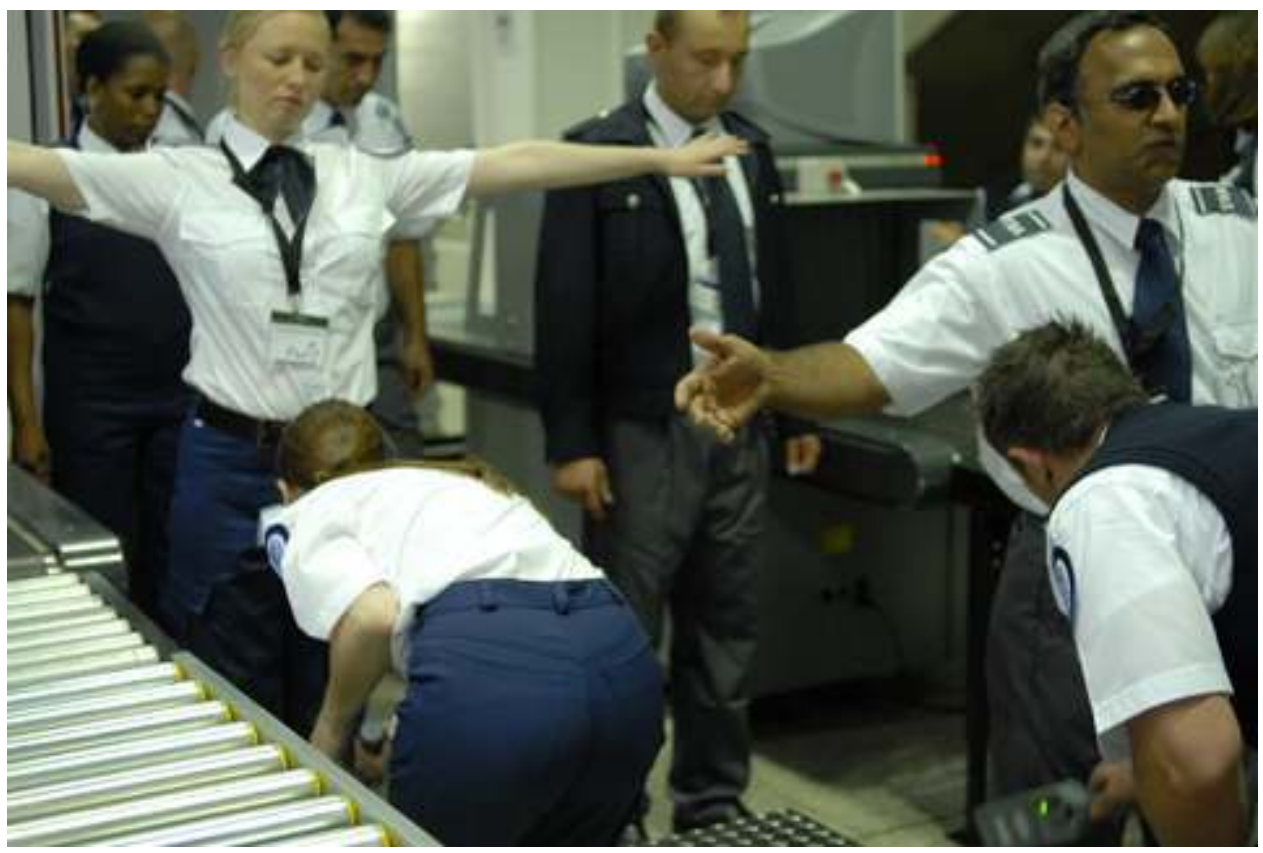

Fig. 3 : Aernout Mik, Touch, rise and fall, 2008, two channel video installation, video still, Courtesy carlier I gebauer, Berlin

8 Touch, rise and fall (2008) présente en continu des images familières pour tout voyageur habitué aux aéroports. Elle est en effet centrée sur les opérations de contrôle auxquelles chacun doit se soumettre pour accéder aux halls de départ. Dans ces sas d'entrée, corps et objets sont séparés pour être scannés et palpés. Partout des mains s'activent pour tour à tour trier, manipuler et diriger sacs, manteaux, ordinateurs d'un côté et passagers de l'autre. Le cadrage se concentre sur les mains, sur les actions de saisie, de palpage ou 
simplement de participation au bon fonctionnement du dispositif de contrôle. La caméra est toujours en mouvement, se déplaçant lentement, se rapprochant, multipliant les points de vue, comme si elle cherchait elle aussi à se saisir tactilement du réel. Les images d'Aernout Mik relèvent véritablement ici d'une vision haptique. Le toucher est ainsi pointé dans son rôle au sein de l'organisation de la surveillance, répondant à l'état de méfiance généralisé qui structure aujourd'hui les sociétés.

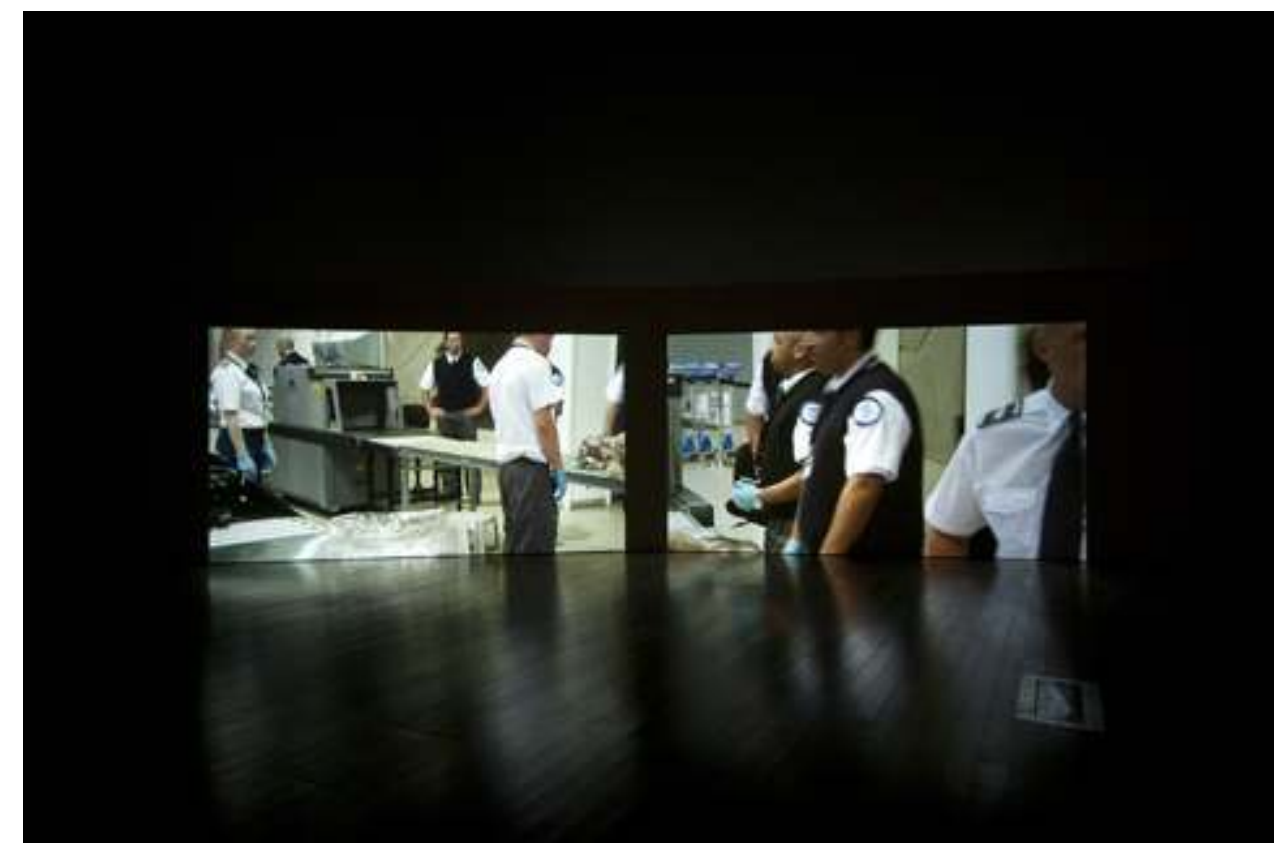

Fig. 4 : Aernout Mik, Touch, rise and fall, 2008, 2 screen video installation, digital video on hard drive, installation view at National Art Center, Tokyo, 2010, courtesy carlier I gebauer

9 L'installation Touch, rise and fall est une large projection associant les images de deux caméras, créant des ruptures et des faux raccords qui complexifient la lecture. Le spectateur peut vite être désemparé face à une progression linaire régulièrement perturbée par des images récurrentes et des temporalités dissonantes. Comme l'explique Elie During, ce type d'œuvre joue de la déconnexion, de la coupure, offrant l'expérience temporelle de la dislocation ${ }^{4}$.

On connaît bien le rôle de la vision dans la surveillance grâce aux analyses de Michel Foucault dans Surveiller et Punir (1975) qui prend le cas de l'organisation de la ville pestiférée pour montrer comment elle structure les états occidentaux: «La ville pestiférée, toute traversée de hiérarchie, de surveillance, de regard, d'écriture, la ville immobilisée dans le fonctionnement d'un pouvoir extensif qui porte de façon indistincte sur tous les corps individuels - c'est l'utopie de la cité parfaitement gouvernée $»^{5}$. Le parallèle avec la ville réagissant aux menaces terroristes est évident : en réponse aux attentats, les gouvernements décrètent des états d'urgence qui, normalisés, permettent de bafouer les libertés individuelles. Chacun est fiché, les déplacements sont surveillés, les zones privées des existences sont pénétrées. Mais tout se passe essentiellement à travers le regard : la surveillance pose le primat du visuel sur le tactile, et part du principe qu'il s'agit de rendre visible toutes les strates des activités constituant une société. Le modèle du panoptique proposé par Jérémy Bentham (philosophe britannique du XVIIIe siècle) et analysé par Michel Foucault, est à la base de la gestion actuelle de la surveillance à partir d'une position omnisciente du regard. En organisant des pénitenciers, des asiles, des hôpitaux et des écoles où chacun est isolé mais objet de 
regard en permanence, on évite les complots, les contagions, les copiages. «La foule, masse compacte, lieu d'échanges multiples, individualités qui se fondent, effet collectif, est abolie au profit d'une collection d'individualités séparées. Du point de vue du gardien, elle est remplacée par une multiplicité dénombrable et contrôlable; du point de vue des détenus, par une solitude séquestrée et regardée $»^{6}$. Le détenu se sait être en tout instant possiblement observé et participe ainsi implicitement à cette surveillance généralisée. Le tour de force de l'état moderne est dans l'intégration par les individus de toutes ces règles, dans l'assimilation des structures de contrôle. A travers l'importance accordée à ces organisations panoptiques, il s'agit aussi d'éviter que les corps se touchent, d'éviter les foules compactes. Plus globalement, la société se construit sur une mise à distance du toucher au profit du visuel.

Néanmoins, et c'est là un aspect que l'œuvre d'Aernout Mik met en évidence, malgré les nouvelles technologies, malgré les outils de vision virtuels qui ont permis d'accroître la surveillance du réel, d'enregistrer tous les mouvements et déplacements (carte bancaire, puces des téléphones), le toucher est encore utilisé dans sa dimension la plus archaïque (palper un corps ou un objet avec les mains) mais aussi avec des excroissances technologiques comme les sondes qui permettent de repérer la présence de certains matériaux, par exemple les métaux dans les aéroports. Le contraste est fort entre la technologie de pointe des outils scannant les corps et les objets par une action visuelle, par un regard pénétrant, et le besoin de recourir néanmoins au sens du toucher pour inspecter autrement, en tâtonnant. Le modèle du panoptique, posé encore aujourd'hui comme base de l'organisation de la surveillance, est questionné par cette place du toucher.

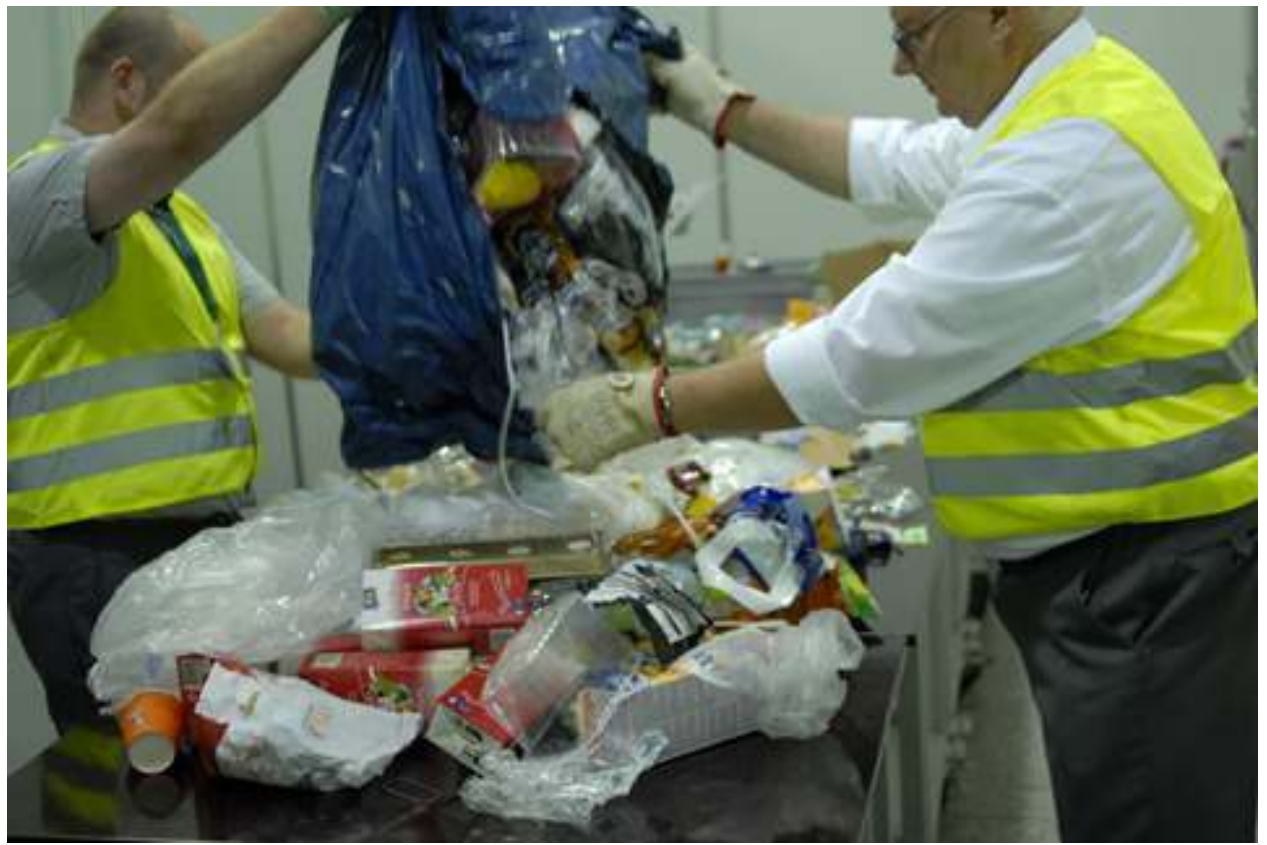

Fig. 5 : Aernout Mik, Touch, rise and fall, 2008, two channel video installation, video still, Courtesy carlier I gebauer, Berlin

Une séquence de l'œuvre se passe dans les magasins duty free de l'aéroport. L'attente dans les aéroports est un temps de consommation, d'achats de dernière minute, compulsifs, d'objets saisis, touchés, reposés, puis repris pour être consommés. Aernout Mik dit ainsi dans un entretien être frappé par la "sensation tactile inséparable de l'achat ${ }^{7}$. Les agents font ici une ronde où ils palpent les peluches, les éventrent parfois, prolongeant 
l'acte du consommateur en donnant au toucher une dimension nettement plus agressive. De même, on les voit à un autre moment inspecter longuement et avec minutie des déchets plastiques, les passant ensuite aux détecteurs à métaux. L'activité de surveillance étendue aux rebuts peut sembler excessive et absurde, et évoquer à l'inverse une forme d'impuissance face à un terrorisme dont les lieux d'ancrage sont diffus et mobiles. Le contrôle frénétique des objets répond au besoin d'avoir une réaction claire et directe au sentiment de menace. Les procédures s'exposent, participant à l'instauration d'un état de méfiance tout en se revendiquant comme instruments de restauration de la confiance. Le contrôle de sécurité est là pour rappeler la menace permanente et sa (bonne) prise en charge par les gouvernements. Aernout Mik nous en montre la violence, symbolique dans les rapports humains, et réelle dans le rapport aux objets. L'expérience est d'autant plus frappante pour le spectateur qu'il en connaît l'inefficacité générale, régulièrement mise en cause par les médias qui en rapportent les failles nombreuses.

La réactivation du toucher dans cette gestion de la surveillance est peut-être finalement surtout le signe d'un échec. C'est parce que les machines les plus efficaces peuvent être trompées, parce que les systèmes les plus pointus sont régulièrement mis en défaut que l'on revient à une forme ancestrale de la surveillance à travers le toucher, redonnant un rôle à l'humain. Mais c'est aussi parce que les scanners corporels soulèvent encore des résistances à leur mise en place systématique. Pourtant, comme le rappelle le site des aéroports français, tout cela est mis en place pour notre sécurité :

"Soumettez-vous de bonne grâce aux contrôles de sûreté : il y va de votre propre intérêt ! Ces contrôles sont effectués avant l'arrivée en salle d'embarquement. Les bagages à mains doivent passer dans un appareil de contrôle radioscopique. Sur un écran, l'agent de sûreté visualise leur contenu. Vos bagages à main peuvent également faire l'objet d'une inspection visuelle ou d'une visite manuelle $»^{8}$.

La terminologie utilisée va dans le sens du primat du visuel («radioscopique», " visualise ») et il est intéressant que la mention du tactile soit exprimée par l'idée d'une "visite », terme étymologiquement relié à la vue, et bien plus attentif à l'intégrité de l'autre que ne le laisse entendre celui d' « inspection ». 


\section{À mains nues}

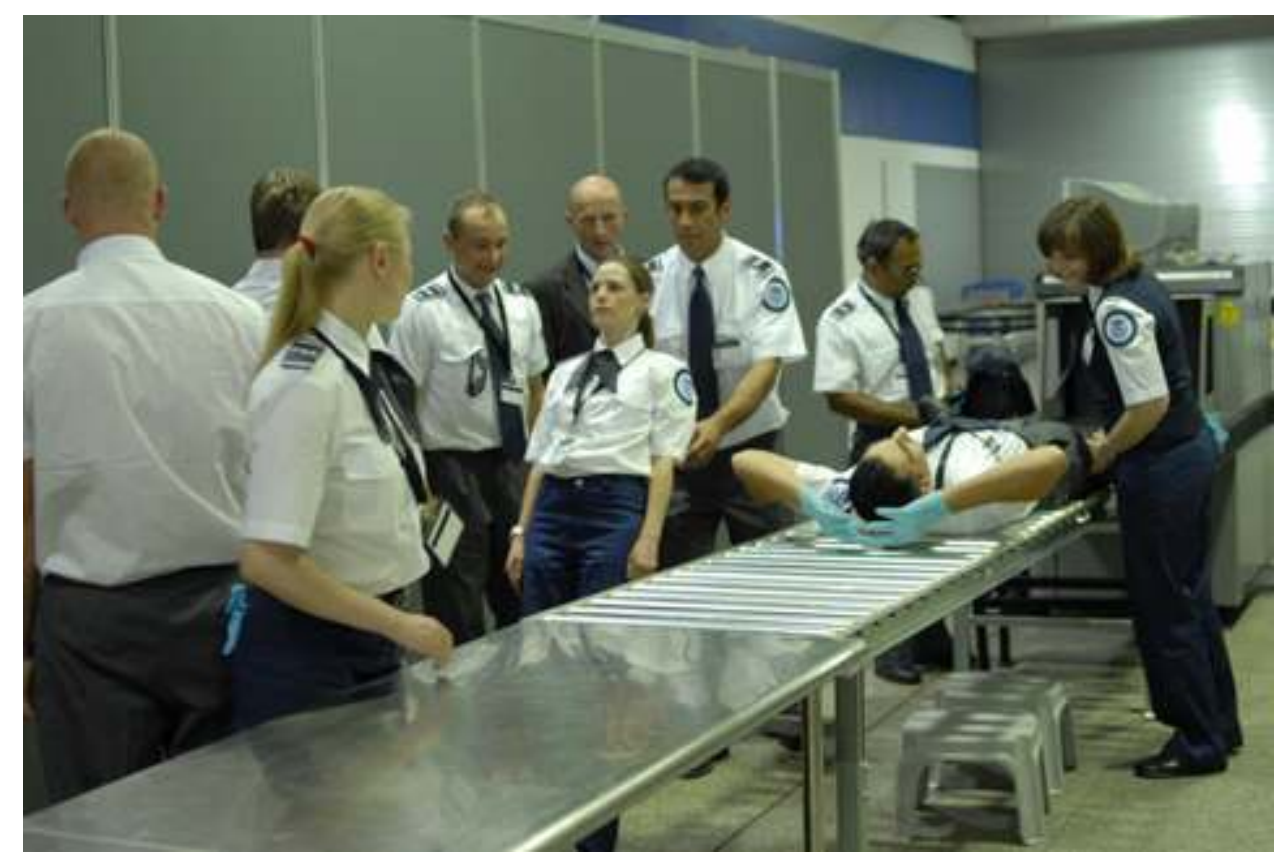

Fig. 6 : Aernout Mik, Touch, rise and fall, 2008, two channel video installation, video still, Courtesy carlier I gebauer, Berlin

Comme dans les architectures panoptiques, Aernout Mik met en évidence la relation au corps individuel qui est isolé pour être inspecté et fouillé. Lorsque son tour arrive, le passager est exclu de la foule, sans être accueilli dans son identité personnelle mais considéré d'emblée comme terroriste en puissance, et isolé à ce titre. Les agents au travail sont également enfermés dans leur fonction qui les met à distance. Aernout Mik a filmé à l'opposé de longues séquences pendant les pauses du personnel, qui cette fois discutent joyeusement, se massent, se divertissent, et semblent alors faire groupe, contrastant fortement avec les passagers qui, bien que présents en masse, sont fondamentalement seuls au moment de leur passage. En filmant les agents dans ces activités ludiques, Aernout Mik montre comment ils ré-apprivoisent leur propre corps et les corps des autres. Se heurter ou se bagarrer sont avant tout des moyens de retrouver contact avec son corps, tout comme le massage, ou les jeux. Le détournement des tapis roulants est particulièrement évocateur puisque cet outil du contrôle devient alors un instrument de relaxation. Le jeu de la chute, où une personne se laisse tomber en arrière, est aussi très symbolique d'un état de confiance retrouvée. Dans le temps de la pause s'entrevoit ainsi l'atmosphère de communitas, d'un vivre-ensemble fait de paroles, d'échanges de regards, de retrouvailles avec son corps propre et de place faite au corps de l'autre.

Ces moments de jeux rappellent d'une certaine manière les rituels qui, comme l'a montré Victor Tuner à propos de la tribu des Ndembu en Zambie, ont une fonction sociale?. Ils permettent de rejouer et libérer sur un terrain symbolique des tensions et conflits qui resteraient irrésolus puisque les Ndembu n'ont pas d'organisation politique, et donc ni de règles ni de lois. Aernout Mik évoque lors de cette scène inattendue avec les agents un état collectif débarrassé de certaines barrières corporelles, où les énergies négatives accumulées seraient expulsées à travers des jeux choisis pour leur manière de mettre en scène des relations individuelles. Pour autant, il ne s'agit pas ici de croire en une possible 
amélioration des conditions de travail par des méthodes qui rappellent également certaines études de "coaching». Si celles-ci ne sont guère efficaces, c'est qu'elles s'appliquent à des individus qui ne vivent pas dans un état communautaire et ne partagent pas de croyances collectives. Les jeux sont ici utilisés pour leur pouvoir suggestif, rappelant la capacité du toucher à mettre en relation des corps dans un contexte autre. Le toucher participe alors d'expressions de confiance, ne cherchant pas à traquer le mal mais à construire un espace commun. Cette fois, les agents n'ont plus de gants et s'investissent dans l'échange à mains nues. Ils prennent le risque du contact et construisent les conditions de la rencontre, qui nécessite de toucher en regardant, en s'adressant à l'autre. Un acte du toucher qui s'associe aussi à une distance, contrairement à Organic Escalator ou Glutinosity, où les corps se touchent mais ne se parlent pas. Les corps amassés les uns aux autres, immergés dans le monde sans distanciation perdent leur capacité à affirmer leur humanité ${ }^{10}$. Dans ce projet de vivre-ensemble, il s'agit de ne pas s'installer dans la fusion et confusion des corps et des regards mais dans des gestes qui s'adressent, qui véhiculent une intention, une relation, et qui laissent ensuite la place au regard. Comme Marie-José Mondzain le décrit dans son analyse d'une trace de main dans la grotte préhistorique de Chauvet, il est nécessaire de creuser un écart pour construire un regard, de poser la main puis de l'enlever : « Produire des images, c'est inscrire dans le visible avec son corps, ici avec ses mains et avec sa bouche, des opérateurs de séparation et donc d'altérité $»^{11}$. Le couple toucher et séparer est la condition d'une construction politique où l'individu s'adresse à l'autre dans son humanité.

Dans le cas des installations vidéo, l'expérience des images est indétachable du lieu où elles adviennent, ce qu'Aernout Mik a bien compris puisqu'il accorde une grande importance aux scénographies de ses expositions. Dans Communitas au Jeu de Paume, il avait ainsi conçu des cimaises anormalement basses permettant au spectateur d'apercevoir l'ensemble de l'exposition, de circuler entre les cimaises-écrans sans isoler les œuvres, alternant entre différents modes de regard, du balayement globalisant à la fixation concentrée. L'ensemble de l'exposition était en outre baigné dans le silence puisqu'aucune vidéo n'avait de bande-son. Ce parti-pris fort d'Aernout Mik accentuait le sentiment d'irréalité qui se dégage de certaines de ses pièces, et fonctionnait également comme un outil de séparation. L'atmosphère moins hypnotique engageait le spectateur dans une circulation, l'éloignant de l'attention fascinée que procure le cinéma pour le conduire dans un cheminement chaotique, fait d'une pluralité de regards, distraits parfois, médusés souvent, mais jamais complètement absorbés. A distance des images, il pouvait ainsi vivre une expérience forte tout en faisant face au monde, en y questionnant sa place en tant que corps sentant mais mobile, présent à l'espace et aux autres dans une errance tâtonnante.

\section{NOTES}

1. Communitas est également le titre d'une installation vidéo datant de 2010 et présentée dans l'exposition. 
2. «Ce sont comme si elles étaient réduites ou rabaissées à une condition uniforme pour être rabaissé à nouveau ", Victor W. Turner, The Ritual Process (1969), trad. fr. Le Phénomène rituel. Structure et contre-structure, Paris, P.U.F, 1990

3. Ce que l'on retrouve par exemple dans le récit qu'un personnage de Don Delillo fait de sa descente de la tour du World Trade Center le 11 septembre 2011 dans L'homme qui tombe, Paris, Actes Sud, 2008, pp. 69-74.

4. Elie During, Faux raccord, Actes Sud/ Villa Arson, 2010.

5. Michel Foucault, Surveiller et Punir, Gallimard, 1975, p. 232.

6. Michel Foucault, ibid. p. 234.

7. Aernout Mik dans un entretien avec Coromandel Brombacker, Spits, 6 janvier 2003.

8. Texte publié sur le site des Aéroports français, http://www.aeroport.fr/passager/surete-etsecurite.php, consulté en mai 2013.

9. «Le rituel ndembu, dans sa forme originelle, avec sa richesse en symboles multivoques (ou polysémiques), peut être considéré comme un instrument qui parvient merveilleusement à exprimer, à maintenir et à épurer périodiquement l'ordre social séculaire, lequel, dépourvu d'une forte centralisation politique, est générateur de conflits multiples", Les tambours d'affliction. Analyse des rituels chez les Ndembu de Sambie, Victor W. Turner, Paris, NRF Gallimard, 1972, p.32.

10. «Si l'homme ne doit pas être enfermé dans la gangue du milieu syncrétique où l'animal vit comme en état d'extase, s'il doit avoir conscience d'un monde comme raison commune de tous les milieux et théâtre de tous les comportements, il faut qu'entre lui-même et ce qui appelle son action s'établisse une distance ", Maurice Merleau-Ponty, Phénoménologie de la perception, Paris, Tel Gallimard, 1945, p. 116.

11. Marie-José Mondzain, Homo spectator, Paris, Bayard, 2007, p. 25.

\section{RÉSUMÉS}

L'oeuvre d'Aernout Mik (artiste néerlandais) filme des corps en interrogeant leur manière d'être présent à un lieu et aux autres, et revient régulièrement sur le motif du toucher. L'installation vidéo Touch, Rise and Fall (2008) s'attache plus particulièrement à montrer ce qui se joue dans les procédures de contrôle dans les aéroports, pour ceux qui en font l'épreuve mais aussi pour ceux qui en adoptent les gestes. Les modes de surveillance des états modernes ont imposé le primat de la vision comme outil de contrôle, mettant à distance les corps et les regards. Pourtant, c'est bien le toucher qui est au coeur des images d'Aernout Mik, engageant une réflexion sur les modes de rapport entre les individus contemporains, entre contact, confiance et séparation.

\section{AUTEUR}

\section{MATHILDE ROMAN}

Docteur en arts et sciences de l'art de l'Université Paris 1 et enseigne au Pavillon Bosio, Ecole supérieure d'art et de scénographie de Monaco. Elle est l'auteur de deux essais : On stage. La dimension scénique de l'image vidéo (LE GAC PRESS, 2012) et Art vidéo et mise en scène de soi (L'Harmattan, 2008). Elle fait partie de l'Institut ACTE), laboratoire de l'Université Paris 1 et du 
groupe de recherche « Le parasitage comme stratégie artistique, brouillage et perturbation des appareillages », MSH Paris Nord et Université Rennes 2. Elle a publié dans les revues Ligeia, Raison Publique et collabore régulièrement comme critique d'art (AICA) à différentes revues (Mouvement, Zérodeux, Art Press, lacritique.org) et catalogues d'exposition. Elle organise aussi projections et conférences autour de vidéos d'artistes, dont le festival Vidéo'val (Université de Créteil / Macval). 